\title{
Estudo da Decomposição de Plantas Aquáticas em Função das Quantidades Depositadas, Da UMidade e do Tipo De Disposição No SOLO $^{1}$
}

\author{
Study on Aquatic Plant Decomposition Due to Amount Deposited, Moisture and Type of \\ Placement in Soil
VELINI, E.D. ${ }^{2}$, CORREAA, M.R. ${ }^{3}$, NEGRISOLI, E. ${ }^{3}$, BRAVIN, L.F.N. ${ }^{4}$, CAVENAGHI, A.L. ${ }^{5}$, ROSSI, C.V.S. ${ }^{3}$ e SILVA, J.R.M. ${ }^{6}$

\begin{abstract}
RESUMO - Um estudo foi realizado com a finalidade de avaliar a decomposição da biomassa de plantas aquáticas, incorporadas ou não ao solo, provenientes do controle mecânico, no reservatório da UHE Americana. O ensaio foi realizado em casa de vegetação, localizada no Núcleo de Pesquisas Avançadas em Matologia (NUPAM) da FCA/Unesp-Botucatu. A avaliação foi conduzida em vasos contendo $14 \mathrm{~kg}$ de solo, simulando descartes de 50,100, $150 \mathrm{e}$ 200 t MF de plantas ha ${ }^{-1}$ e avaliando o processo de decomposição através da liberação de $\mathrm{CO}_{2}$, divididos em duas etapas: a primeira em solo seco e, a segunda, na seqüência, com o solo úmido. A quantificação do $\mathrm{CO}_{2}$ liberado foi realizada através de titulação de solução adicionada ao processo de incubação de 24 horas dos vasos. Os dados foram interpolados e analisados seguindo modelo de Mitscherlich, com algumas modificações. Na primeira etapa, foi observada uma rápida liberação de $\mathrm{CO}_{2}$ até o décimo dia, seguida de estabilização. $\mathrm{O}$ maior teor de $\mathrm{CO}_{2}$ liberado foi observado no tratamento com descarte de $200 \mathrm{t} \mathrm{MF} \mathrm{ha}^{-1}$ incorporado ao solo. Os dados avaliados durante a segunda fase do ensaio representaram uma maior linearidade no processo de liberação de $\mathrm{CO}_{2}$, indicando um período mais longo do processo de degradação da biomassa descartada.
\end{abstract}

Palavras-chave: macrófitas aquáticas, degradação, liberação de $\mathrm{CO}_{2}$

\begin{abstract}
A study was carried out to evaluate biomass decomposition of aquatic macrophytes incorporated or not into soil, due to mechanical control, at the Americana/SP UHE Reservoir. Analysis was performed in a greenhouse at the Weed Science Research Center (NUPAM), UNESP, Botucatu. Plastic vases containing $24 \mathrm{~kg}$ of soil were assessed by simulating discards of 50, 100, 150 and $200 t$ of fresh matter ha ${ }^{-1}$.The decomposition process was evaluated by $\mathrm{CO}_{2}$ release, divided into two stages: dry soil and wet soil. Quantification of released $\mathrm{CO}_{2}$ was performed through titration of solution added to vase incubation. Data were interpolated and analyzed by Mitscherlich model with some modifications. A quick $\mathrm{CO}_{2}$ release up to the $10^{\text {th }}$ day was observed in the first stage (dry soil), followed by stabilization. The highest $\mathrm{CO}_{2}$ content released was

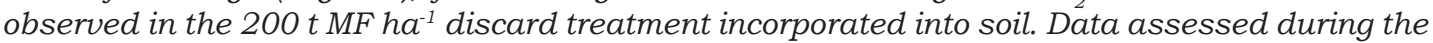
second stage (wet soil) showed a higher linearity in the $\mathrm{CO}_{2}$ release process, indicating a longer period of discarded biomass decomposition.
\end{abstract}

Key words: aquatic macrophytes, degradation, $\mathrm{CO}_{2}$ release.

1 Recebido para publicação em 5.1.2005 e na forma revisada em 6.4.2005.

Trabalho financiado pela linha de P\&D, ANEEL.

2 Prof. Assistente Dr., Departamento de Produção Vegetal/Agricultura, FCA/UNESP, Caixa Postal 237, 18603-970 Botucatu- SP, <velini@fca.unesp.br>; ${ }^{3}$ Eng.-Agr., M.S., Programa de pós-graduação em Agricultura, Dep. de Produção Vegetal/Agricultura, FCA/UNESP, Botucatu-SP. ${ }^{4}$ Eng. Naval, M.S., Prof. Faculdade de Tecnologia de Botucatu - FATEC, Botucatu-SP. ${ }^{5}$ Eng.-Agr., D.S., Dep. de Produção Vegetal/Agricultura, FCA/UNESP, Botucatu-SP. ${ }^{6}$ Graduando em Tecnologia em Gestão de Sistema de Informação, Faculdade Marechal Rondon - FMR, 18650-000 São Manuel-SP. 


\section{INTRODUÇÃO}

As macrófitas aquáticas são importantes componentes de lagos, rios e reservatórios (Asaeda et al., 2000), pois constituem fonte de oxigênio, alimento e abrigo para a vida aquática (Velini, 2000; Miyazaki \& Pitelli, 2003). Entretanto, a grande carga de esgotos residenciais e industriais favorece a eutrofização desses ambientes aquáticos, com conseqüente desequilíbrio, caracterizado pela disponibilidade de nutrientes, acelerando assim o crescimento da vegetação aquática indesejável (Greenway, 1997; Bitar, 1998).

Quando o desenvolvimento dessas plantas é intenso, acarreta prejuízos consideráveis, com grandes massas desses vegetais dificultando a navegação, pesca, recreação e entupindo tomadas de água das turbinas de usinas hidrelétricas. A partir desse momento, tornase necessário o seu controle.

Algumas são as alternativas para o controle dessas espécies: o método químico, à base de produtos de ação herbicida, como diquat, glyphosate, 2,4-D e fluridone (Rimer, 1984; Martins, 1998); o biológico, utilizando espécies de peixes, mamíferos, insetos, ácaros e caramujos (Miyazaki \& Pitelli, 2003); e o mecânico, com o uso de embarcações e equipamentos apropriados, com a finalidade de retirada da biomassa do corpo hídrico e descarte em ambiente terrestre (Velini, 2000).

No que se refere ao controle mecânico, apesar de ele apresentar algumas vantagens em relação aos demais, pela não-contaminação da água com componentes químicos ou tóxicos, algumas desvantagens podem ser citadas. A inespecificidade da operação causa danos à fauna e flora não-daninha, além da possibilidade de segmentação das plantas com propagação vegetativa, os quais são aspectos importantes a serem considerados.

No entanto, quando as plantas são transportadas até a margem, há a preocupação adicional em descartá-las sem que problemas ambientais sejam gerados. Conforme relatado por Velini (2000), as tentativas de se criar um uso para a biomassa a ser descartada têm sido infrutíferas, em razão das quantidades envolvidas e do elevado teor de água dessas plantas.

Planta Daninha, Viçosa-MG, v. 23, n. 2, p. 249-254, 2005
Com base nessas informações, foi realizado um estudo com o objetivo de avaliar a degradação de biomassa de plantas aquáticas no solo, em grandes quantidades, provenientes de práticas de controle mecânico no reservatório da UHE Americana/SP.

\section{MATERIAL E MÉTODOS}

O estudo foi realizado em casa de vegetação, no Núcleo de Pesquisas Avançadas em Matologia (NUPAM) da FCA/UNESP - Botucatu, e constituiu-se em um experimento com delineamento inteiramente casualizado, com nove tratamentos e quatro repetições. O estudo foi desenvolvido em duas fases: a primeira consistiu em estudar a liberação de $\mathrm{CO}_{2}$ das plantas em solo seco, utilizando-se somente a umidade presente nas plantas. Após a estabilização do processo, iniciou-se uma segunda fase, em que se adicionava água em quantidade preestabelecida, e assim sucessivamente, quando necessário, por meio de avaliação visual do solo.

Amostras compostas de Eichhornia crassipes, Pistia stratiotes e Brachiaria subquadripara, coletadas no reservatório da UHE Americana, nas quantidades totais de 50, 100, 150 e 200 t matéria fresca ha-1 , foram $^{-1}$ acondicionadas em vasos plásticos com capacidade de $14 \mathrm{~kg}$ de solo, representando área de $0,0888 \mathrm{~m}^{2}$. Quatro tratamentos consistiram de incorporação desse material ao solo, em quatro tratamentos manteve-se a massa fresca em cobertura no solo, e uma testemunha continha apenas solo. Após o processo de incorporação e acomodação da biomassa fresca nos vasos, iniciou-se o processo de determinação de liberação de $\mathrm{CO}_{2}$ proveniente da degradação do referido material.

Para quantificação do $\mathrm{CO}_{2}$ liberado, em cada vaso foi colocado um frasco contendo $20 \mathrm{~mL}$ de $\mathrm{NaOH}$ a $1 \mathrm{~N}$, para retenção do gás. Depois disso, todos os vasos foram lacrados e incubados no escuro, em temperatura média de $25^{\circ} \mathrm{C}$. A leitura do $\mathrm{CO}_{2}$ liberado foi feita 24 horas após a incubação, sendo o solo mantido em repouso por um período mínimo de 48 horas entre uma incubação e outra.

Posteriormente ao período de incubação, os frascos contendo $\mathrm{NaOH}$ foram retirados para titulação. Inicialmente foram adicionadas à 
solução cinco gotas de solução alcoólica de fenolftaleína a $0,5 \%$, titulada com $\mathrm{HCl}$ a 0,65 $\mathrm{N}$. Após a primeira viragem, foram adicionadas cinco gotas de solução alcoólica de dimetilorange a $0,1 \%$. Quando a segunda viragem ocorria, era anotada a diferença de volume de $\mathrm{HCl}$ entre as duas viragens. Deve ser destacado que, entre as duas viragens ( $\mathrm{pHs}$ de aproximadamente 8,3 e 6,7), todo o $\mathrm{CO}_{2}$ encontrava-se na forma de $\mathrm{HCO}_{3}^{-}$. A quantificação do $\mathrm{CO}_{2}$ liberado pôde ser calculada pela seguinte expressão:

$$
\mathrm{gCO}_{2} \text { liberado }=\mathrm{N}_{e q} \mathrm{CO}_{2} \times 44
$$

em que $\mathrm{N}_{\text {eq }} \mathrm{CO}_{2}=V_{\text {(titulado) }}(L) \times 0,65$.

O valor obtido pela expressão corresponde a g de $\mathrm{CO}_{2}$ liberado vaso ${ }^{-1}$, posteriormente convertido a $\mathrm{kg} \mathrm{CO}_{2}$ liberado $\mathrm{ha}^{-1}$.

Os dados da titulação para quantificação de $\mathrm{CO}_{2}$ liberado no processo de respiração foram interpolados, para que pudessem ser comparados a cada dia, durante todo o período de avaliação. Esses dados foram analisados seguindo o modelo de Mitscherlich. Entretanto, para que isso ocorresse, necessitou-se modificá-lo. Os modelos original e adaptado podem ser observados a seguir:

$$
\begin{aligned}
& \text { Original }-Y=A x\left(1-10^{-C x(X+B)}\right) \\
& \text { Adaptado }-Y=A x\left(1-10^{-C x X}\right)
\end{aligned}
$$

em que $Y=\mathrm{CO}_{2}$ liberado ajustado, em $\mathrm{kg} \mathrm{ha}^{-1}$; $A=$ assintota horizontal máxima do modelo original; $C=$ concavidade da curva; e $X=\mathrm{CO}_{2}$ liberado original, em $\mathrm{kg} \mathrm{ha}^{-1}$.

Com a finalização das avaliações dessa fase, deu-se início à segunda fase de avaliação do ensaio, com a adição de água no sistema em quantidade equivalente a uma chuva de $30 \mathrm{~mm}$. Em seguida, no decorrer do ensaio, a umidade do solo foi monitorada visualmente e, quando necessário, foi adicionada uma quantidade equivalente à chuva de $10 \mathrm{~mm}$, mantendo-se a umidade do solo em torno de $60 \%$ da capacidade de campo. O processo de avaliação da liberação de $\mathrm{CO}_{2}$ a seguir foi o mesmo utilizado na primeira fase, com periodos de incubação, titulação e avaliação do comportamento degradativo das plantas.

Os dados da titulação para quantificação de $\mathrm{CO}_{2}$ liberado no processo de respiração, durante esta fase, foram interpolados, para serem comparados a cada dia, durante a avaliação. Para ajuste dos dados, estes foram analisados seguindo o modelo de Mitscherlich.

\section{RESULTADOS E DISCUSSÃO}

\section{Primeira Fase}

Os resultados referentes à liberação de $\mathrm{CO}_{2}$ nos diferentes tratamentos, durante a primeira fase, podem ser observados nas Figuras 1 a 4, em que são comparados individualmente os respectivos tratamentos mantidos em solo seco.

Para avaliação da liberação de $\mathrm{CO}_{2}$ com a quantidade descartada de $50 \mathrm{t} \mathrm{MF} \mathrm{ha}^{-1}$ (Figura 1), tanto o tratamento com biomassa incorporada como aquele com biomassa mantida em superfície apresentaram comportamentos praticamente idênticos, com ligeira superioridade para o material incorporado ao solo, com acúmulo total de $291 \mathrm{~kg} \mathrm{CO}_{2} \mathrm{ha}^{-1}$ durante o período de avaliação.

Quando se avaliou a quantidade descartada de $100 \mathrm{t} \mathrm{ha}^{-1}$ (Figura 2), foi observada similaridade no comportamento do processo degradativo nas duas condições. O tratamento com material incorporado apresentou maior liberação de $\mathrm{CO}_{2}$ desde o início do ensaio, com liberação total acumulada de $412 \mathrm{~kg} \mathrm{ha}^{-1}$, durante os 33 dias avaliados. Esse resultado indica que, para essa quantidade de biomassa descartada e nessa condição de solo, a degradação do material incorporado mostrou-se mais acelerada e eficiente.

Avaliando a degradação para os tratamentos com 150 t ha $^{-1}$ (Figura 3), observou-se o contrário do visto anteriormente. Neste caso, a biomassa mantida na superficie do solo liberou mais $\mathrm{CO}_{2}$, fazendo com que o processo degradativo fosse mais acentuado em comparação à testemunha e ao tratamento com material incorporado ao solo. A quantidade de $\mathrm{CO}_{2}$ liberada e acumulada para este tratamento ficou em torno de $556 \mathrm{~kg} \mathrm{ha}^{-1}$, enquanto a testemunha liberou $250 \mathrm{~kg} \mathrm{ha}^{-1}$.

Ao avaliar a quantidade de $\mathrm{CO}_{2}$ liberada nos tratamentos com descarte de $200 \mathrm{t} \mathrm{MF} \mathrm{ha}^{-1}$, percebeu-se uma inversão no comportamento deles (Figura 4). Observou-se que o tratamento em que a biomassa foi mantida na superficie

Planta Daninha, Viçosa-MG, v. 23, n. 2, p. 249-254, 2005 


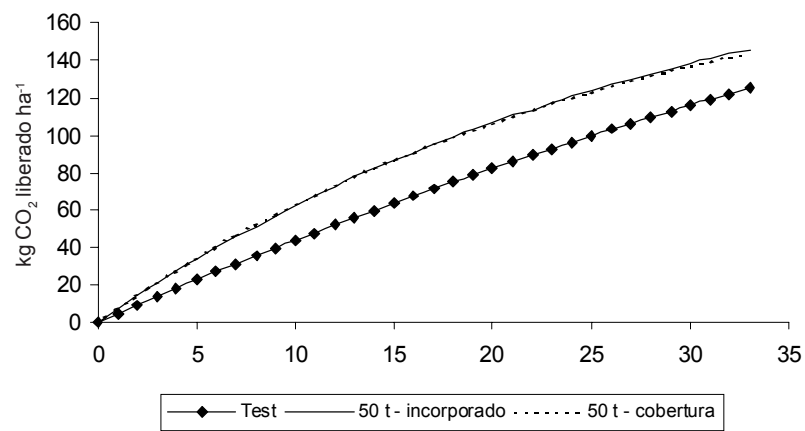

Parâmetros de Mitscherlich: Testemunha ( $\mathrm{A}=348,0244$; $\left.\mathrm{C}=0,00585 ; \mathrm{R}^{2}=0,99\right)$

$50 \mathrm{t} \mathrm{ha}^{-1}$ incorporado $\left(\mathrm{A}=214,7507 ; \mathrm{C}=0,01490 ; \mathrm{R}^{2}=0,99\right)$

$50 \mathrm{t} \mathrm{ha}^{-1}$ em cobertura $\mathrm{A}=206,3582 ; \mathrm{C}=0,01574 ; \mathrm{R}^{2}=0,99$ )

Figura 1 - Liberação acumulada de $\mathrm{CO}_{2}\left(\mathrm{~kg} \mathrm{ha}^{-1}\right)$ durante a primeira fase de avaliação, com plantas incorporadas e mantidas em cobertura no solo. Tratamentos com descarte de 50 t MF ha-1. Botucatu-SP, 2004.

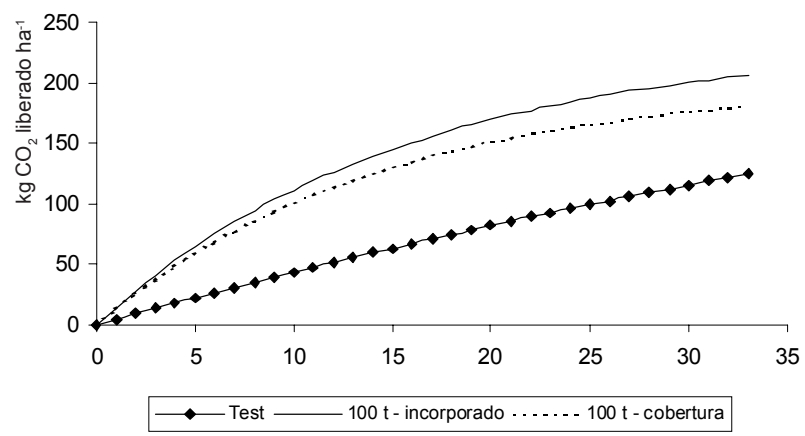

Parâmetros de Mitscherlich: Testemunha (A = 348,0244; $\left.\mathrm{C}=0,00585 ; \mathrm{R}^{2}=0,99\right)$

$100 \mathrm{t} \mathrm{ha}^{-1}$ incorporado $\left(\mathrm{A}=234,1296 ; \mathrm{C}=0,02806 ; \mathrm{R}^{2}=0,99\right)$

$100 \mathrm{t} \mathrm{ha}^{-1}$ em cobertura $\left(\mathrm{A}=202,2261 ; \mathrm{C}=0,03006 ; \mathrm{R}^{2}=0,99\right)$

Figura 2 - Liberação acumulada de $\mathrm{CO}_{2}\left(\mathrm{~kg} \mathrm{ha}^{-1}\right)$ durante a primeira fase de avaliação, com plantas incorporadas e mantidas em cobertura no solo. Tratamentos com descarte de 100 t MF ha $^{-1}$. Botucatu-SP, 2004.

do solo apresentou rápida ascensão na liberação de $\mathrm{CO}_{2}$ e degradação inicial até a estabilização do processo a partir do décimo dia. Ao contrário, quando as plantas foram incorporadas ao solo, esse processo de rápida estabilização não ocorreu, fazendo com que, ao final da avaliação, liberasse maior quantidade de $\mathrm{CO}_{2}$ : em torno de $740 \mathrm{~kg} \mathrm{ha}^{-1}$.

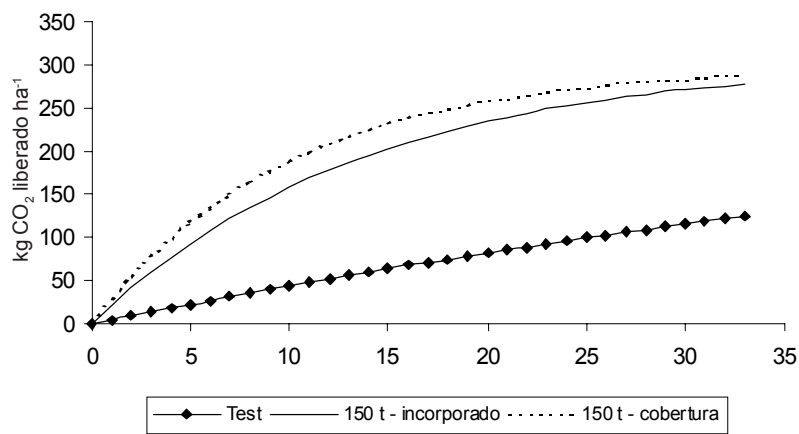

Parâmetros de Mitscherlich: Testemunha (A=348,0244; $\left.\mathrm{C}=0,00585 ; \mathrm{R}^{2}=0,99\right)$

$150 \mathrm{t} \mathrm{ha}^{-1}$ incorporado $\left(\mathrm{A}=305,7298 ; \mathrm{C}=0,03169 ; \mathrm{R}^{2}=0,99\right)$

$150 \mathrm{t} \mathrm{ha}^{-1}$ em cobertura $\left(\mathrm{A}=299,0735 ; \mathrm{C}=0,04345 ; \mathrm{R}^{2}=0,99\right)$

Figura 3 - Liberação acumulada de $\mathrm{CO}_{2}\left(\mathrm{~kg} \mathrm{ha}^{-1}\right)$ durante a primeira fase de avaliação, com plantas incorporadas e mantidas em cobertura no solo. Tratamentos com descarte de 150 t MF ha $^{-1}$. Botucatu-SP, 2004.

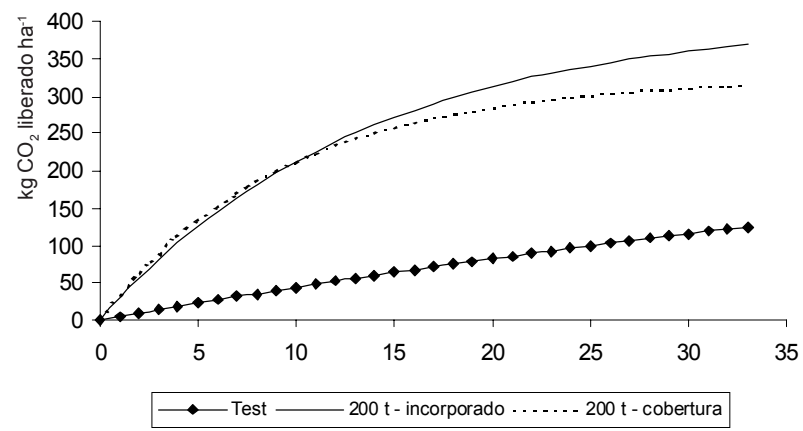

Parâmetros de Mitscherlich: Testemunha (A = 348,0244; $\left.\mathrm{C}=0,00585 ; \mathrm{R}^{2}=0,99\right)$

$200 \mathrm{t} \mathrm{ha}^{-1}$ incorporado $\left(\mathrm{A}=404,0560 ; \mathrm{C}=0,03230 ; \mathrm{R}^{2}=0,99\right)$

$200 \mathrm{tha}^{-1}$ em cobertura $\left(\mathrm{A}=323,8792 ; \mathrm{C}=0,04620 ; \mathrm{R}^{2}=0,99\right)$

Figura 4 - Liberação acumulada de $\mathrm{CO}_{2}\left(\mathrm{~kg} \mathrm{ha}^{-1}\right)$ durante a primeira fase de avaliação, com plantas incorporadas e mantidas em cobertura no solo. Tratamentos com descarte de 200 t MF ha $^{-1}$. Botucatu-SP, 2004.

Pôde ser observada em todas as avaliações a rápida liberação inicial de $\mathrm{CO}_{2}$, até o décimo dia, seguida de estabilização a partir desse momento. A maior quantidade de $\mathrm{CO}_{2}$ liberada foi observada no tratamento com descarte e incorporação no solo de $200 \mathrm{t} \mathrm{MF} \mathrm{ha-1,}$ representando duas vezes e meia a observada com a menor quantidade (50 t $\mathrm{MF} \mathrm{ha}^{-1}$ ). 


\section{Segunda Fase}

Os resultados referentes à liberação de $\mathrm{CO}_{2}$ nos diferentes tratamentos, durante a segunda fase (com adição de água no sistema), podem ser observados nas Figuras 5 a 8, em que são comparados individualmente os respectivos tratamentos mantidos em solo com umidade em torno de $60 \%$.

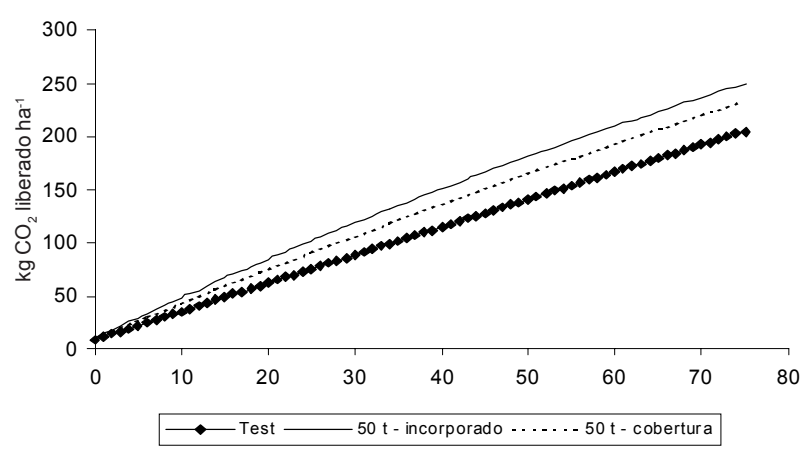

Parâmetros de Mitscherlich: Testemunha $(\mathrm{A}=3070,0526$; $\left.\mathrm{B}=3,1159 ; \mathrm{C}=0,00038 ; \mathrm{R}^{2}=0,99\right)$

$50 \mathrm{t} \mathrm{ha}^{-1}$ incorporado $(\mathrm{A}=658,2125 ; \mathrm{B}=2,5417 ; \mathrm{C}=0,00253$, $\left.\mathrm{R}^{2}=0,99\right)$

$50 \mathrm{t} \mathrm{ha}^{-1}$ em cobertura $(\mathrm{A}=1004,3546 ; \mathrm{B}=2,8980 ; \mathrm{C}=0,00147$; $\left.\mathrm{R}^{2}=0,99\right)$

Figura 5 - Liberação acumulada de $\mathrm{CO}_{2}\left(\mathrm{~kg} \mathrm{ha}^{-1}\right)$ durante a segunda fase de avaliação, com plantas incorporadas e mantidas em cobertura no solo. Tratamentos com descarte de 50 t MF ha $^{-1}$. Botucatu-SP, 2004

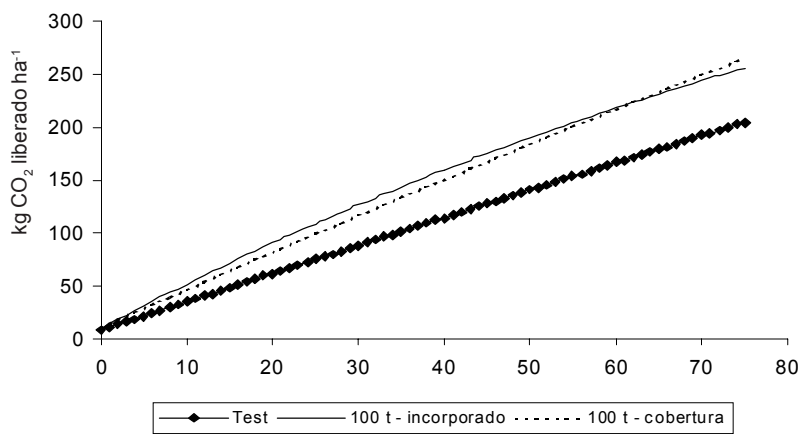

Parâmetros de Mitscherlich: Testemunha $(\mathrm{A}=3070,0526$; $\left.\mathrm{B}=3,1159 ; \mathrm{C}=0,00038 ; \mathrm{R}^{2}=0,99\right)$

$100 \mathrm{t} \mathrm{ha}^{-1}$ incorporado $(\mathrm{A}=548,8360 ; \mathrm{B}=2,1478 ; \mathrm{C}=0,00354$, $\left.\mathrm{R}^{2}=0,99\right)$

$100 \mathrm{t} \mathrm{ha}^{-1}$ em cobertura $(\mathrm{A}=2290,5770 ; \mathrm{B}=3,1464 ; \mathrm{C}=0,00068$; $\left.\mathrm{R}^{2}=0,99\right)$

Figura 6 - Liberação acumulada de $\mathrm{CO}_{2}\left(\mathrm{~kg} \mathrm{ha}^{-1}\right)$ durante a segunda fase de avaliação, com plantas incorporadas e mantidas em cobertura no solo. Tratamentos com descarte de 100 t MF ha $^{-1}$. Botucatu-SP, 2004.
$\mathrm{Na}$ avaliação dos tratamentos com descarte de $50 \mathrm{t} \mathrm{ha}^{-1}$ (Figura 5), o processo degradativo foi mais intenso quando estas estavam incorporadas ao solo, correspondendo a um acréscimo de aproximadamente $7 \%$ de $\mathrm{CO}_{2}$ liberado em relação ao tratamento comparativo.

Observou-se semelhança entre os valores obtidos e o comportamento dos tratamentos

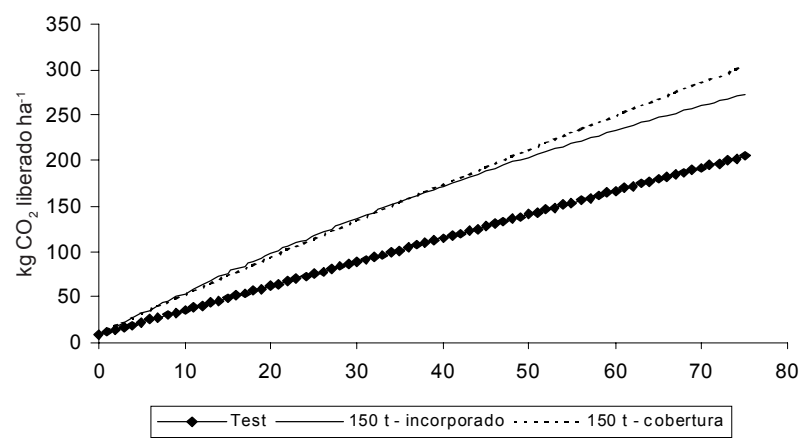

Parâmetros de Mitscherlich: Testemunha (A = 3070,0526; $\left.\mathrm{B}=3,1159 ; \mathrm{C}=0,00038 ; \mathrm{R}^{2}=0,99\right)$

$150 \mathrm{t} \mathrm{ha}^{-1}$ incorporado $(\mathrm{A}=528,4762 ; \mathrm{B}=1,4293 ; \mathrm{C}=0,00412$, $\left.\mathrm{R}^{2}=0,99\right)$

$150 \mathrm{t} \mathrm{ha}^{-1}$ em cobertura $(\mathrm{A}=1675,5261 ; \mathrm{B}=2,2363$; $\left.\mathrm{C}=0,00113 ; \mathrm{R}^{2}=0,99\right)$

Figura 7 - Liberação acumulada de $\mathrm{CO}_{2}\left(\mathrm{~kg} \mathrm{ha}^{-1}\right)$ durante a segunda fase de avaliação, com plantas incorporadas e mantidas em cobertura no solo. Tratamentos com descarte de 150 t MF ha $^{-1}$. Botucatu-SP, 2004.

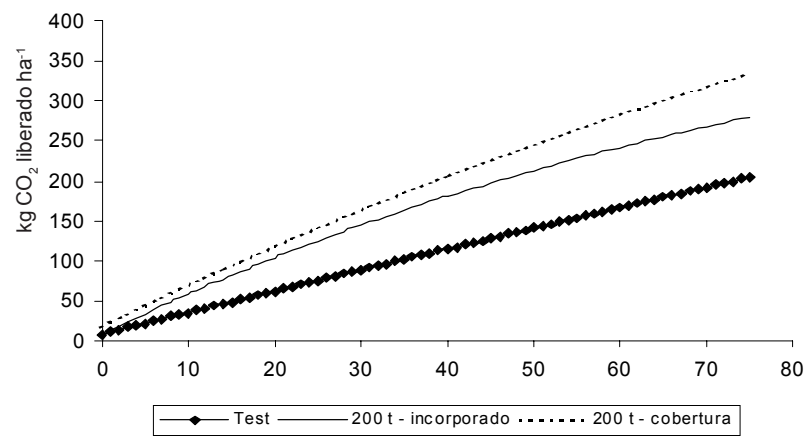

Parâmetros de Mitscherlich: Testemunha $(\mathrm{A}=3070,0526$; $\mathrm{B}=$ 3,$\left.1159 ; \mathrm{C}=0,00038 ; \mathrm{R}^{2}=0,99\right)$

$200 \mathrm{t} \mathrm{ha}^{-1}$ incorporado $(\mathrm{A}=478,3021 ; \mathrm{B}=1,3060 ; \mathrm{C}=0,00499$; $\left.\mathrm{R}^{2}=0,99\right)$

$200 \mathrm{t} \mathrm{ha}^{-1}$ em cobertura $(\mathrm{A}=823,0193 ; \mathrm{B}=3,2033 ; \mathrm{C}=0,00289$; $\left.\mathrm{R}^{2}=0,99\right)$

Figura 8 - Liberação acumulada de $\mathrm{CO}_{2}\left(\mathrm{~kg} \mathrm{ha}^{-1}\right)$ durante a segunda fase de avaliação, com plantas incorporadas e mantidas em cobertura no solo. Tratamentos com descarte de 200 t MF ha $^{-1}$. Botucatu-SP, 2004.

Planta Daninha, Viçosa-MG, v. 23, n. 2, p. 249-254, 2005 
com descarte de 100 t MF ha $^{-1}$ (Figura 6) e $150 \mathrm{t} \mathrm{MF} \mathrm{ha}^{-1}$ (Figura 7). Houve pequena diferença entre os tratamentos, tanto no manejo da biomassa quanto nas quantidades a serem descartadas. A quantidade de $\mathrm{CO}_{2}$ liberada e acumulada durante o período avaliado de 75 dias oscilou entre 510 e $608 \mathrm{~kg} \mathrm{CO}_{2} \mathrm{ha}^{-1}$.

Resultado expressivo foi verificado para a quantidade descartada de 200 t ha $^{-1}$ (Figura 8), em que a degradação da biomassa em cobertura do solo foi mais intensa, chegando à liberação e acúmulo de aproximadamente $670 \mathrm{~kg} \mathrm{CO} \mathrm{ha}^{-1}$, enquanto o tratamento com biomassa incorporada ao solo gerou cerca de $560 \mathrm{~kg} \mathrm{CO} \mathrm{ha}^{-1}$, durante essa segunda fase de avaliação.

Diferentemente do que foi observado na primeira fase do ensaio, constatou-se maior linearidade no processo de liberação de $\mathrm{CO}_{2}$, indicando um período mais longo do processo de degradação da biomassa descartada.

\section{AGRADECIMENTOS}

À CPFL, Companhia Paulista de Força e Luz, pela colaboração e pelo apoio técnico no desenvolvimento deste trabalho.

\section{LITERATURA CITADA}

ASAEDA, T.; TRUNG, V. K.; MANTUNGE, J. Modeling the effects of macrophyte growth and decomposition on the nutrient budget in Shallow Lakes. Aquatic Bot., v. 68, p. 217-237, 2000.

BITAR, A. L. Fluxo de nitrogênio e seu uso por duas macrófitas (E. crassipes e $P$. stratiotes) no reservatório de Salto Grande (Americana-SP). 1998. 97 f. Dissertação (Mestrado em Ciências da Engenharia Ambiental Universidade de São Paulo, São Carlos, 1998.

GREENWAY, M. Nutrient content of wetland plants in constructed wetlands receivng municipal effluent in tropical Australia. Wat. Sci. Technol., v. 35, n. 5, p. 135-142, 1997.

MARTINS, D. Controle de plantas daninhas aquáticas. In: WORKSHOP CONTROLE DE PLANTAS AQUÁTICAS, 1998, Brasília. Resumos... Brasília: IBAMA, 1998. p. 30-31.

MIYAZAKI, D. M. Y.; PITELLI, R. A. Estudo do potencial do pacu (Piaractus mesopotamicus) como agente de controle biológico de Egeria densa, E. najas e Ceratophyllum demersum. Planta Daninha, v. 21, p. 53-59, 2003. (Edição especial)

RIMER, D. N. Introduction to freshwater vegetation. London: AVI Publishing, 1984. 207 p.

VELINI, E. D. Controle de plantas aquáticas. In: CONGRESSO BRASILEIRO DA CIÊNCIA DAS PLANTAS DANINHAS, 22., 2000, Foz do Iguaçu. Palestras... Foz do Iguaçu: SBCPD, 2000. p. 137-147. 